Original article

\title{
A comparison of clinical outcomes between vaccinated and vaccine-naive patients of COVID-19, in four tertiary care hospitals of Kerala, South India
}

\author{
Sabarish Balachandran ${ }^{\mathrm{a}}$, Merlin Moni ${ }^{\mathrm{b}}$, Dipu T. Sathyapalan ${ }^{\mathrm{b}}$, Prinoj Varghese ${ }^{\mathrm{c}}$, \\ Manoj P. Jose ${ }^{\mathrm{d}}$, Mithun R. Murugan ${ }^{\mathrm{e}}$, C. Rajan ${ }^{\mathrm{c}}$, Dhanraj Saboo ${ }^{\mathrm{f}}$, Sooraj S. Nair ${ }^{\mathrm{g}}$, \\ Reshmi Ann Varkey ${ }^{h}$, Parvathy Balachandran ${ }^{i}$, Geetha R. Menon ${ }^{j}$, Beena K. Vasudevan ${ }^{\mathrm{h}}$, \\ Amitava Banerjee $^{\mathrm{k}}$, Chandrasekhar Janakiram ${ }^{\mathrm{i}}$, Jaideep C. Menon ${ }^{1, *}$ \\ a Department of Emergency Medicine, Amrita Institute of Medical Sciences, Amrita Vishwa Vidyapeetham, Kochi, Kerala, 682041, India \\ ${ }^{\mathrm{b}}$ Division of Infectious Diseases, Amrita Institute of Medical Sciences, Amrita Vishwa Vidyapeetham, Kochi, Kerala, 682041, India \\ ${ }^{\mathrm{c}}$ Department of General Medicine, Sree Narayana Institute of Medical Sciences, Kerala, 683594, India \\ d Department of General Medicine, Little Flower Hospital and Research Centre, Kerala, 683572, India \\ e Internal Medicine, PRS Hospital, Thiruvananthapuram, Kerala, 695002, India \\ ${ }^{\mathrm{f}}$ Department of Medicine, Little Flower Hospital and Research Centre, Kerala, India \\ ${ }^{g}$ PRS Hospital, Killipalam, Thiruvananthapuram, Kerala, 695002, India \\ ${ }^{\mathrm{h}}$ Department of Public Health, Amrita Institute of Medical Sciences, Amrita Vishwa Vidyapeetham, Kochi, Kerala, 682041, India \\ ${ }^{\mathrm{i}}$ Amrita School of Dentistry, Amrita Institute of Medical Sciences, Amrita Vishwa Vidyapeetham, Kochi, Kerala, 682041, India \\ ${ }^{\mathrm{j}}$ ICMR- National Institute of Medical Statistics, New Delhi, India \\ ${ }^{\mathrm{k}}$ Professor in Clinical Data Science and Honorary Consultant Cardiologist, Institute of Health Informatics, University College London, United Kingdom \\ ${ }^{1}$ Preventive Cardiology Unit, Amrita Institute of Medical Sciences, Amrita Vishwa Vidyapeetham, Kochi, Kerala, 682041, India
}

\section{A R T I C L E I N F O}

\section{Keywords:}

COVID-19

Vaccine

Coronavirus

Vaccine-breakthrough infection

Outcomes

\begin{abstract}
A B S T R A C T
The problem considered: This multi-centric study analyzed data of COVID-19 patients and compared differences in symptomatology, management, and outcomes between vaccinated and vaccine-naive patients.

Methods: All COVID-19 positive individuals treated as an in-or out-patient from the $1^{\text {st }}$ March to $15^{\text {th }}$ May 2021 in four selected study sites were considered for the study. Treatment details, symptoms, and clinical course were obtained from hospital records. Chi-square was used to test the association of socio-demographic and treatment variables with the vaccination status and binary logistic regression were used to obtain the odds ratio with a $95 \%$ confidence interval.

Results: The analysis was of 1446 patients after exclusion of 156 with missing data of which males were $57.3 \%$ and females $42.7 \%$. 346 were vaccinated; 189 received one dose and 157 both doses. Hospitalization was more in vaccinated (38.2\% vs $27.4 \%)$; ICU admissions were less in vaccinated (3.5\% vs $7.1 \%)$. More vaccinated were symptomatic $(\mathrm{OR}=1.5)$; half less likely to be on non-invasive ventilation $(\mathrm{OR}=0.5)$ while vaccine naive patients had 4.21 times the risk of death.

Conclusion: Severe infection, duration of hospital stays, need for ventilation and death were significantly less among vaccinated when compared with vaccine naive patients.
\end{abstract}

\section{Introduction}

Health systems worldwide have been overwhelmed by the speed and extent of the spread of the pandemic with several countries reporting excessive mortality both directly due to COVID-19 and collaterally in unrelated disease areas as well. ${ }^{2,3}$ Deaths due to COVID-19 have largely been in individuals above the age of 60 and those with other co-morbid conditions, with COVID-19 infection being seen as a trigger to events leading to death. ${ }^{1,6}$

Starting from December of 2020 vaccines against COVID-19 were made available in various parts of the globe. There are at least 13 vaccines across four different platforms presently in use globally. ${ }^{4}$ Of these seven vaccines have seen widespread use across countries and it is estimated that there are 150 other types of vaccines in various stages of

\footnotetext{
* Corresponding author.

E-mail address: menon7jc@gmail.com (J.C. Menon).
} 
development or efficacy testing. The two most widely used vaccines in India are the COVISHIELD developed by AstraZeneca/Oxford University and being manufactured by the Serum Institute of India (SII) ${ }^{2}$ and the COVAXIN(3) developed indigenously in India by the Indian Council of Medical Research (ICMR) and Bharat Biotech. COVISHIELD is a recombinant, replication-deficient chimpanzee adenovirus vector encoding the SARS-CoV-2 Spike (S) glycoprotein produced in genetically modified human embryonic kidneys (HEK) 293 cells, ${ }^{5}$ while COVAXIN has been developed using the whole-virion inactivated Vero cell-derived platform technology. ${ }^{6}$

As with all viruses especially RNA viruses the likelihood of mutations increases with an increase in infection or transmission of disease. There are many variants of the original SARS-COV-2 (alphavirus) in transmission including the delta, beta, and kappa variants. Each of the variants is different from the original in either of transmissibility, infectivity, and severity of infection. Mutations in the receptor-binding motif (RBM), the site where the spike protein (S protein) binds to the ACE2 receptor is of special interest to virologists as this can potentially result in non-recognition of $S$ protein by antibodies generated secondary to vaccination especially with the S-protein platform vaccines. ${ }^{7}$

There have been two waves of infection in the continuing pandemic, the first between June and October 2020 and the second between March and June 2021. In the second wave, the case-fatality rate (CFR) has been higher with more ICU admissions, more need for ventilator care, and need for oxygen as compared to the first wave. The transmissibility of the second wave was also noted to be more with almost all family members getting infected in a household.

A vaccine breakthrough infection is defined as the detection of SARSCoV-2 RNA or antigen in a respiratory specimen collected from a person $\geq 14$ days after they have completed all recommended doses of an authorized COVID-19 vaccine. The myriad reasons due to which vaccine breakthrough could arise include mutations in the virus, ineffectiveness of the vaccine secondary to various factors including a break in the cold chain, etc, faulty techniques of vaccination, and host factors leading to ineffective antibody production. ${ }^{4,5}$

Vaccine breakthrough infection was first reported from the US where a total of 10262 SARS-CoV-2 vaccine breakthrough infections had been reported from 46 U.S. states and territories as of April 30, 2021. Among these cases, 6446 (63\%) occurred in females, and the median patient age was 58 years. Based on preliminary data, 2725 (27\%) vaccine breakthrough infections were asymptomatic, 995 (10\%) patients were known to be hospitalized, and 160 (2\%) patients died. Among the 995 hospitalized patients, $289(29 \%)$ were asymptomatic or hospitalized for a reason unrelated to COVID-19. The median age of patients who died was 82 years (interquartile range $71-89$ years). ${ }^{6}$

A study from Israel suggested that variants of concern (VOCs) were able to elude the host antibody response generated from the vaccine. ${ }^{7}$ In a study done in 7080 fully vaccinated healthcare workers (HCWs) from Christian Medical College, Vellore, the investigators reported the protective effect of vaccination in preventing infection, hospitalization, need for oxygen, and ICU admission as 65\%, 77\%, 92\%, and $94 \%$ respectively. ${ }^{8}$ In another study from PGIMER, Chandigarh the investigators reported the incidence of breakthrough infection as 1.6\% (48 of $3000 \mathrm{HCWs}$ ); the median time from receipt of the second dose to breakthrough infection was 29.5 days (interquartile range, 20 to 35). ${ }^{9}$

\section{Study objectives}

To compare outcomes between vaccinated and vaccine naive COVIDpositive patients treated in four tertiary hospitals in Kerala, South India.

\section{Methods}

This is a retrospective study that was conducted at four tertiary care hospitals treating COVID-19 patients in Kerala, India. The study was approved by the Institutional Ethics Committee (IEC No ECASM-AIMS-
2021-254, dated $18^{\text {th }}$ May 2021). Data of both in-patients and outpatients during the period of March 1, 2021, till May 15, 2021, were obtained from COVID-19 wards, Intensive Care Units, and COVID registers of the study sites. The data obtained comprised of details regarding treatment, symptoms, and course in the hospital. COVID-19 positive individuals who were examined at the study centres and managed with domiciliary care/FLTC were contacted telephonically. In case of incomplete medical records, vaccination status was confirmed through telephone.

Data of all COVID-19 positive patients who agreed to participate in the study during the study period (March 1, 2021-May 15, 2021) were included. Non - consenting COVID-19 patients and COVID-19 positive patients with missing data that could not be completed through telephone follow-up were excluded from the study.

Data extracted for analysis comprised of age, gender, place of treatment-domiciliary, FLTC, hospital or Intensive care, date of test positivity, duration of hospital stay if admitted, dates of vaccination, organs involved-respiratory, cardiac, gastrointestinal, neurologic (including headache), treatment - anticoagulants, antivirals, steroids, antimicrobials, oxygen, invasive or non-invasive ventilation and post COVID-19 symptoms at 1 week after discharge. Individuals were considered to have clinically mild infection if they did not need supplemental oxygen during their disease course.

The extracted data collected from four centres was entered on a Microsoft Excel spreadsheet and was analyzed using SPSS 27 version. Descriptive analyses for categorical variables were expressed in frequencies and proportions. A Chi-square test was used for comparing the vaccinated to the vaccine-naive groups for various treatment and infection parameters. The association of socio-demographic and clinical parameters with the vaccination status was expressed as odds ratio (OR) with $95 \%$ confidence intervals. A p-value of 0.05 was considered statistically significant.

\section{Results}

Records of 1602 patients were available of which 156 with missing values were excluded from the analysis, thereby, resulting in1446 records. Among the 1446 patients, 828 (57.3\%) were males and 618 $(42.7 \%)$ were females. Based on the patient status, $63.8 \%(n=923)$ patients were managed as domiciliary care or in first-line treatment centres (FLTC), 29.9\% $(n=433)$ as in-patients and 6.2\% $(n=90)$ were admitted in intensive care (ICU). Most patients were non-vaccinated (n $=1100,76.1 \%$ ), while of the 346 individuals who were vaccinated, 189 received one dose while 157 had received both doses of the vaccine.

The vaccinated group were characterised by males (50.7\%), 15-45 age group $(50.9 \%)$, domiciliary care $(56.6 \%)$, symptomatic $(81.8 \%)$ with fever (54\%), respiratory $(49.1 \%)$, gastrointestinal $(6.9 \%)$, neurological including headache(22\%), cardiac (1.4\%) symptoms, invasive fungal infection (1.2\%); drugs used-steroids (9.2\%), antivirals (11.5\%), anticoagulants $(82.9 \%)$, antimicrobials $(54.9 \%)$,oxygen $(7.5 \%)$, noninvasive ventilation $(4.3 \%)$, mechanical ventilation $(5.2 \%)$, average length of hospital stay (8.06+- 4.18 days) and death (1.4\%). In the vaccine naïve cohort 65 patients(65/1100,5.9\%) required mechanical ventilation as compared to 18 patients(18/328,5.4\%)in the vaccinated cohort.

Comparing between the vaccinated and the vaccine naive COVID-19 patients, ICU admissions* (3.5\% vs $7.1 \%$ ), fever ( $58.5 \%$ vs $54 \%$ ), gastrointestinal symptoms ( $9.5 \%$ vs $6.9 \%$ ), non-invasive ventilation use (10\% vs $4.3 \%)^{*}$, death $(5.8 \%$ vs $1.4 \%) *$, mechanical ventilation $(5.9 \%$ vs $5.2 \%$ ),oxygen use ( $9 \%$ vs $7.5 \%$ ), antibiotics (19\% vs $12.4 \%$ ), use of steroids ( $12.8 \%$ vs $9.2 \%)$, antivirals (16\% vs $11.5 \%$ ) and length of stay in hospital $11.33+9.9$ days for the vaccine naive and $8.06+-4.18$ days in the vaccinated group were all favourable for the vaccinated cohort with*ICU admissions, need for non-invasive ventilation and death reaching statistical significance.

Table 1(Table 1 near here) shows that the vaccinated group has a 
Table 1

Association of treatment parameters and outcomes with vaccination status.

\begin{tabular}{|c|c|c|c|c|c|c|c|c|c|}
\hline \multirow[t]{2}{*}{ Variables } & & \multicolumn{2}{|c|}{ Vaccinated } & \multicolumn{2}{|c|}{ Non-Vaccinated } & \multirow[t]{2}{*}{$\mathrm{p}$ value } & \multirow[t]{2}{*}{ Odds Ratio } & \multicolumn{2}{|c|}{$95 \%$ CI } \\
\hline & & $\mathrm{n}$ & $\%$ & $\mathrm{n}$ & $\%$ & & & LCI & UCI \\
\hline \multirow[t]{3}{*}{ Age (in years) } & $46-60$ & 69 & 24.0 & 218 & 76.0 & 0.294 & 1.18 & 0.86 & 1.62 \\
\hline & $>61$ & 98 & 31.6 & 212 & 68.4 & 0.001 & $1.73^{*}$ & 1.29 & 2.31 \\
\hline & $<45$ & 179 & 21.1 & 670 & 78.9 & & & & \\
\hline \multirow[t]{2}{*}{ Gender } & Female & 171 & 27.7 & 447 & 72.3 & 0.004 & $1.43^{*}$ & 1.12 & 1.82 \\
\hline & Male & 175 & 21.1 & 653 & 78.9 & & & & \\
\hline \multirow{3}{*}{ Patient status } & IP & 132 & 30.5 & 301 & 69.5 & 0.001 & $1.57^{*}$ & 1.21 & 2.02 \\
\hline & ICU & 12 & 13.3 & 78 & 86.7 & 0.058 & 0.55 & 0.29 & 1.03 \\
\hline & Domiciliary/Covid Care centres & 202 & 21.9 & 721 & 78.1 & & & & \\
\hline \multirow{2}{*}{ COVID symptoms } & Yes & 283 & 25.6 & 824 & 74.4 & 0.008 & $1.50^{*}$ & 1.11 & 2.04 \\
\hline & No & 63 & 18.6 & 276 & 81.4 & & & & \\
\hline \multirow[t]{2}{*}{ Fever } & Yes & 187 & 22.5 & 643 & 77.5 & 0.148 & 0.84 & 0.66 & 1.07 \\
\hline & No & 159 & 25.8 & 457 & 74.2 & & & & \\
\hline \multirow[t]{2}{*}{ Respiratory symptoms } & Yes & 170 & 28.3 & 431 & 71.7 & 0.001 & $1.50^{*}$ & 1.18 & 1.91 \\
\hline & No & 176 & 20.8 & 669 & 79.2 & & & & \\
\hline \multirow[t]{2}{*}{ Abdominal symptoms } & Yes & 24 & 18.6 & 105 & 81.4 & 0.137 & 0.71 & 0.45 & 1.12 \\
\hline & No & 322 & 24.4 & 995 & 75.6 & & & & \\
\hline \multirow[t]{2}{*}{ Neurological symptoms } & Yes & 76 & 36.7 & 131 & 63.3 & 0.001 & $2.08^{*}$ & 1.52 & 2.85 \\
\hline & No & 270 & 21.8 & 969 & 78.2 & & & & \\
\hline \multirow{2}{*}{ Cardiac involvement } & Yes & 5 & 25.0 & 15 & 75.0 & 0.909 & 1.06 & 0.38 & 2.94 \\
\hline & No & 341 & 23.9 & 1085 & 76.1 & & & & \\
\hline \multirow[t]{2}{*}{ Another organ involvement } & Yes & 64 & 27.6 & 168 & 72.4 & 0.154 & 1.26 & 0.92 & 1.73 \\
\hline & No & 282 & 23.2 & 932 & 76.8 & & & & \\
\hline \multirow[t]{2}{*}{ Invasive fungal infection/medication } & Yes & 4 & 28.6 & 10 & 71.4 & 0.686 & 1.27 & 0.40 & 4.08 \\
\hline & No & 343 & 23.9 & 1090 & 76.1 & & & & \\
\hline \multirow[t]{2}{*}{ Steroid medications } & Yes & 32 & 18.5 & 141 & 81.5 & 0.074 & 0.69 & 0.46 & 1.04 \\
\hline & No & 314 & 24.7 & 959 & 75.3 & & & & \\
\hline \multirow[t]{2}{*}{ Anticoagulant medications } & No & 59 & 21.0 & 222 & 79.0 & 0.199 & 0.81 & 0.59 & 1.12 \\
\hline & Yes & 287 & 24.6 & 878 & 75.4 & & & & \\
\hline \multirow[t]{2}{*}{ Antiviral medications } & Yes & 42 & 22.7 & 143 & 77.3 & 0.676 & 0.92 & 0.64 & 1.34 \\
\hline & No & 304 & 24.1 & 957 & 75.9 & & & & \\
\hline \multirow[t]{2}{*}{ Antimicrobial medications } & Yes & 162 & 26.5 & 450 & 73.5 & 0.052 & $1.27^{*}$ & 1.00 & 1.62 \\
\hline & No & 184 & 22.1 & 650 & 77.9 & & & & \\
\hline \multirow[t]{2}{*}{ Length of hospital stay } & $<7$ days & 94 & 24.2 & 295 & 75.8 & 0.898 & 1.02 & 0.78 & 1.34 \\
\hline & $\geq 7$ days & 252 & 23.8 & 805 & 76.2 & & & & \\
\hline \multirow{2}{*}{ Severity of COVID-19 Infection } & Moderate & 26 & 20.8 & 99 & 79.2 & 0.34 & 0.84 & 0.53 & 1.31 \\
\hline & Mild & 1001 & 24.2 & 320 & 75.8 & & & & \\
\hline
\end{tabular}

Vaccination status and vaccine breakthrough

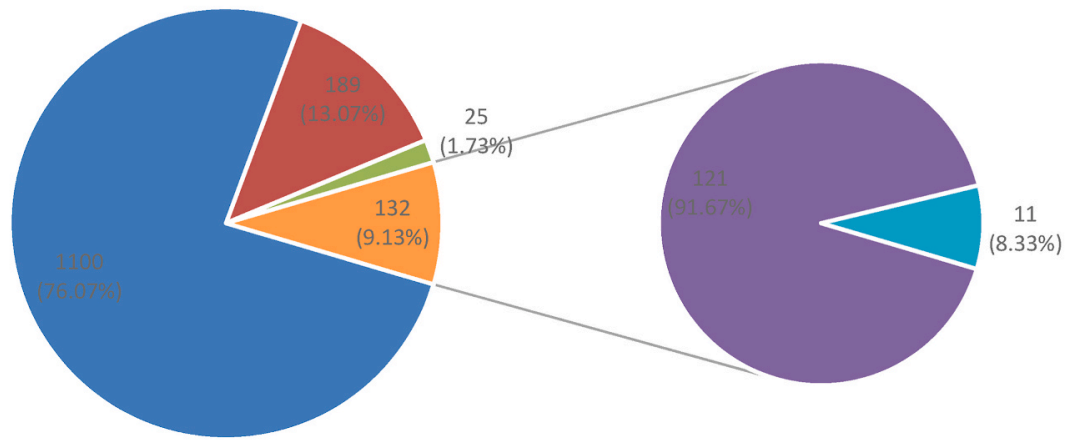

$\begin{array}{ll}\text { - Vaccine naïve } & \text { - First dose only } \\ \text { - Infection before second dose } & \text { - Vaccine breakthrough } \\ \text { - Infection within } 14 \text { days of second dose } & \end{array}$

Fig. 1. Vaccination status and vaccine breakthrough. 
$45 \%$ less ICU admissions ( $\mathrm{OR}=0.55,95 \%$ CI $0.29-1.03$ ) as compared to COVID care centres although not significant. However, vaccine naive has $57 \%$ more risk of ICU admission compared to vaccinated COVID-19 patients ( $\mathrm{OR}=1.57,95 \% \mathrm{CI}=1.21-2.02)$. The vaccinated group was more likely to be symptomatic (OR $=1.5 ; 95 \%$ CI $1.11-2.04$ ) especially with respiratory symptoms (OR $=1.5$; 95\% CI 1.18-1.91); and neurological symptoms (OR $=2.1 ; 95 \%$ CI $1.52-2.85$ ). COVID-19 vaccination reduced the risk of non-invasive ventilation use by $48 \%$ (OR $=0.52$, $95 \% \mathrm{CI}=0.3-0.91$ ) compared to vaccine naive patients. Vaccinated patients reduced the risk of severity by $18 \%$ as compared to vaccine naïve patients though this was not statistically significant (OR $=0.82$; $95 \%$ CI $0.52-1.29$ ). Vaccine naive patients were 4.17 times more likely to succumb to COVID infection than vaccinated patients Of the 346 who had received the vaccination, 214 developed COVID-19 after the first dose of the vaccine, 11 within 14 days of the second dose, and 121 (vaccine-breakthrough) on or after 14 days of the second dose. Of the 69 deaths, 64 were in the vaccine naive patients while there were 5 deaths in the vaccinated: 4 after a single dose of vaccination and only 1 in a vaccine breakthrough infection. (Fig. 1). Deaths occurred in $0.63 \%$ (vaccine breakthrough) of the fully vaccinated, $2.1 \%$ of the partially vaccinated, and in $5.8 \%$ of the vaccine naive infected with COVID-19. Mechanical Ventilation (5.9 \%vs 5.2\%), non-invasive ventilation ( $8 \%$ vs $4.3 \%$ ) and moderate-severe symptoms ( $9 \%$ vs $7.5 \%$ ) were all more favourable for the vaccinated as compared to the vaccine naive (Table 2 near here).

\section{Discussion}

The results of our study comparing symptoms, course, and outcomes between the vaccinated and vaccine naive COVID-19 patients provides us with real-world data on the effectiveness of the available vaccines. Complications due to COVID-19 infection were far less in the vaccinated as compared to the vaccine naive as also the need for anti-viral, antibiotics and steroids. The severity of infection and duration of hospital stay was also less in the vaccinated as compared to the vaccine naive.

Many the vaccinated $<45$ years of age were healthcare workers and hence the disproportion in gender in the vaccinated group. In spite of the number of individuals $>60$ years being more in the vaccinated group $(28$ vs $19 \%$ ) complications were lower in them. The present study reiterates that vaccines help prevent complications of COVID including death.

Analysis of positive cases between the 15 days from October 19th to November 2nd, 2021 by the State Health Department of Kerala ${ }^{10}$ revealed 119041 cases of whom 100593 had been eligible for vaccination of which 67980 (57.9\%) had received one or both doses of the vaccine. Of those eligible for vaccination among the positive cases, 40, 584 (34.9\% of the total count) were fully vaccinated and 27,396 others ( $22.9 \%$ of the total count) had only one dose - the rest were vaccine naive $(43.2 \%)$.

A point-prevalence survey ${ }^{11}$ of almost 100000 people conducted in England in June-July 2021 during the height of that country's spring Delta variant surge found that fully vaccinated people $(n=55962)$ were two-thirds less likely to harbour SARS-CoV-2 compared with unvaccinated people ( $\mathrm{n}=15135$ ), with absolute rates of $0.40 \%$ vs $1.21 \%$, respectively. Likewise, in a randomized trial ${ }^{12}$ of the mRNA-1273 vaccine (Moderna) vs placebo, vaccinated participants $(n=14287$ ) were two-thirds less likely to be asymptomatic carriers than unvaccinated participants ( $\mathrm{n}=14 \mathrm{164}$ ), with absolute rates of $1.5 \%$ vs $3.5 \%$, respectively (estimated vaccine effectiveness against asymptomatic infection, 63.0\% [95\% CI, 56.6\%-68.5\%]).

In a study ${ }^{11}$ of 7771 household contacts of 4921 index cases in the Netherlands, the rate of transmission from fully vaccinated household members was $13 \%$ vs $22 \%$ from unvaccinated household members (estimated vaccine effectiveness against transmission, 63\% [95\% CI, $46 \%-75 \%]$ ). Similarly, in an English study ${ }^{11}$ of 151821 contacts of 99 567 index patients, the rate of transmission from people fully vaccinated with BNT162b2 (Pfizer-BioNTech) was $23 \%$ vs $49 \%$ for transmission
Table 2

Vaccination status and select clinical parameters.

\begin{tabular}{clll}
\hline & $\begin{array}{l}\text { Fully vaccinated } \\
\text { (vaccine } \\
\text { breakthrough) }\end{array}$ & $\begin{array}{l}\text { Partially } \\
\text { vaccinated (either } \\
1 \text { dose or within } 14 \\
\text { days of 2nd dose) }\end{array}$ & $\begin{array}{l}\text { Vaccine } \\
\text { naive }\end{array}$ \\
\hline $\begin{array}{c}\text { No \& (\%) of deaths } \\
\text { according vaccination } \\
\text { status }\end{array}$ & $1 / 157(0.63 \%)$ & $4 / 189(2.11 \%)$ & $\begin{array}{l}64 / 1100 \\
(5.81 \%)\end{array}$ \\
$\begin{array}{c}\text { No \& (\%) of Moderate- } \\
\text { severe COVID-19 } \\
\text { infection according } \\
\text { vaccination status }\end{array}$ & $10 / 157(6.36 \%)$ & $16 / 189(8.5 \%)$ & $\begin{array}{l}99 / 1100 \\
(9 \%)\end{array}$ \\
$\begin{array}{c}\text { No \& (\%) of patients } \\
\text { requiring ventilation } \\
\text { (mechanical and non- } \\
\text { invasive) according to } \\
\text { vaccination status }\end{array}$ & $16 / 157(5.73 \%)$ & $23 / 189(12.12 \%)$ & $74 / 1100$ \\
\hline
\end{tabular}

from unvaccinated people (adjusted odds ratio [aOR], 0.35 [95\% CI, $0.26-0.48]$ for transmission of Delta to unvaccinated contacts; aOR, 0.10 [95\% CI, 0.08-0.13] for transmission of Delta to fully vaccinated contacts). The age-adjusted rate of hospitalization among US adults ${ }^{11}$ aged 18 years or older was 83.6 per 100000 for unvaccinated persons compared with 4.5 per 100000 for fully vaccinated persons.

Further genomic studies are warranted for assessing how many infections especially in cases of vaccine-breakthrough are due to variant strains of the corona virus. The clinical characteristics, hospital course, outcomes both short and long-term are also of special interest in the vaccine-breakthrough infections due to variants.

One of the limitations of our observational study is that it is only from four sites in the state and cannot be called truly representative of the state or country. The study has also not factored for the various laboratory and imaging modalities of individual patients which are used to classify the severity of the COVID-19 illness. The study has not looked at long-term effects of COVID-19 as the period of follow-up was short and limited to one week after discharge or cure.

\section{Conclusion}

This retrospective data analysis of COVID-19 positive patients treated at four tertiary care centres in Kerala, India suggests that severe infection, duration of hospital stay, need for invasive and non-invasive ventilation and death were significantly less in the vaccinated cohort as compared to the vaccine-naive one. Of the five deaths from among the 346 individuals who turned COVID-19 positive after vaccinations, there was only one death in an individual with vaccine-breakthrough infection, while the other four were in individuals after a single dose of vaccine. Vaccines help prevent death and complications secondary to COVID-19 infection.

\section{Declaration of competing interest}

None of the authors have any conflict of interest to declare.

\section{Acknowledgment}

Rohan Prakash, Joslyn Mary Thattil, Sarvesh P, Mani Vignesh K, Sneha Ann John, Sharenya Ashok A, Shruthi Gopinath, Athira Ashok, Aswathy P Nair, Maymol Thoma, Renjith Raja S A for help in gathering and entering data.

\section{References}

1 Elezkurtaj S, Greuel S, Ihlow J, et al. Causes of death and comorbidities in hospitalized patients with COVID-19. Sci Rep. 2021 Feb 19;11(1):4263. 
2 Serum Institute of India - ChAdOx1 nCoV- 19 corona virus vaccine (recombinant) COVISHIELD. Internet, Available from https://www.seruminstitute.com/product_co vishield.php; 2021 Nov 7.

3 COVAXIN - India's first indigenous covid-19 vaccine | Bharat Biotech. Internet, Available from https://www.bharatbiotech.com/covaxin.html; 2021 Nov 7.

4 SARS-CoV-2 variants and vaccines - PubMed. Internet, Available from https://pubm ed.ncbi.nlm.nih.gov/34161052/; 2021 Nov 7.

5 COVID-19 vaccine effectiveness | CDC. Internet, Available from https://www.cdc. gov/coronavirus/2019-ncov/vaccines/effectiveness/index.html?CDC_AA_refVal=h ttps\%3A\%2F\%2Fwww.cdc.gov\%2Fvaccines\%2Fcovid-19\%2Fhealth-departments $\%$ 2Fbreakthrough-cases.html; 2021 Nov 7.

6 Kustin T, Harel N, Finkel U, et al. Evidence for increased breakthrough rates of SARS $\mathrm{CoV}-2$ variants of concern in BNT162b2-mRNA-vaccinated individuals. Nat Med. 2021 Aug;27(8):1379-1384.
7 Protective effect of COVID-19 vaccine among Health care workers during the second wave of the pandemic in India - PubMed. Internet, Available from https://pubmed. ncbi.nlm.nih.gov/34366135/; 2021 Nov 7.

8 Rana K, Mohindra R, Pinnaka L. Vaccine breakthrough infections with SARS-CoV-2 variants. N Engl J Med. 2021 Jul 8;385(2):e7.

9 Philomina JB, Jolly B, John N, et al. Genomic survey of SARS-CoV-2 vaccine breakthrough infections in healthcare workers from Kerala, India. J Infect. 2021 Aug; 83(2):237-279.

10 Internet, cited 2021 Nov 7. Breakthrough Infections Form Chunk of Kerala Covid Cases, Symptoms Mild. The Indian Express; 2021. Available from https://indianexpress. com/article/india/kerala/kerala-covid-19-cases-7607760/.

11 Understanding breakthrough infections following mRNA SARS-CoV-2 vaccination infectious diseases | JAMA | JAMA network. Internet, Available from https:// jamanetwork.com/journals/jama/fullarticle/2786040; 2021 Nov 7.

12 Juthani PV, Gupta A, Borges KA, et al. Hospitalisation among vaccine breakthrough COVID-19 infections. Lancet Infect Dis. 2021 Nov 1;21(11):1485-1486. 